\title{
Designation of Multiple-star Components
}

\author{
Andrei Tokovinin \\ Association of Universities for Research in Astronomy, Cerro Tololo \\ Interamerican Observatory
}

\begin{abstract}
.
Hierarchical multiple stars need designations for individual components, for super-components (several bodies that act as single dynamical center and are often measured jointly) and sub-systems. Hierarchical designations like those proposed for WMC can satisfy all needs, but designations will have to be frozen and will not reflect true hierarchy in the future as new components are discovered. Hence, a clearing house (WMC on the web?) is needed to maintain unique and consistent designations. The hierarchy can be coded separately and flexibly by reference to parent super-components. As an example, these ideas are applied to a real quintuple star HR 1706.
\end{abstract}

\section{What and why designate?}

We are looking for a clear, unique and computer-friendly system for designating multiple stars and planets. We need to designate several different things. First, components - physical bodies like stars or planets. Second, super-components unions of several bodies that act as single dynamical centers. Some observational data (e.g. photometry, astrometry) refer to such combinations measured as a whole, without splitting into true components. Moreover, some components that are now believed to be single will be resolved in the future into sub-systems, so currently available data actually refer to super-components. Finally, we need designations for systems or sub-systems with approximately Keplerian orbits that are the building blocks of more complex stellar or planetary systems. Each system is composed of two components or super-components, which means that designations for systems can be derived from designations of their components. In the following we designate systems by joining two component's designations with comma.

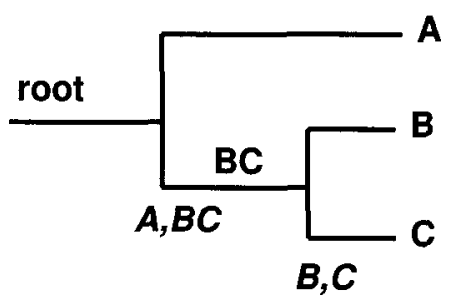

Figure 1. A generic triple system. The components are designated by single letters, super-components - by combinations of letters, systems (in italics) as two components joined by comma. 
The point is illustrated in Fig. 1 showing a simple visual triple where a close pair $\mathrm{BC}$ moves around $\mathrm{A}$ in a wide orbit. The center of gravity of $\mathrm{BC}$ is the secondary in this wide orbit, it is a super-component $\mathrm{BC}$. If $\mathrm{A}$ itself turns to be a spectroscopic binary, then its components would be designated as $\mathrm{Aa}$ and $\mathrm{Ab}$, whereas A becomes a super-component. All measurements of A as a whole will remain valid and there will be no need to change the catalogs.

It is important that designations be fixed and do not change as our knowledge of multiple stars progresses. This conforms to the IAU rules because designations are only a special case of naming astronomical objects. It is desirable to keep existing designations unchanged in order to minimize confusion. So, how should the names for new components be chosen?

\section{Hierarchical or sequential?}

The Washington Multiple Star Catalog (WMC, Hartkopf \& Mason 2003) will adopt component designations that are constructed hierarchically, like Aa and $\mathrm{Ab}$ in the above example. The virtues of hierarchical designations are obvious: 1) super-components are designated by truncation, $\mathrm{Aa} \rightarrow \mathrm{A} ; 2$ ) designations are robust because changes in one sub-tree of a hierarchy do not affect other subtrees; 3 ) designations are meaningful by showing to which (super-)component a given system belongs, thus diminishing chances of confusion. Hierarchical naming is widely used: telephone numbers, internet and postal addresses, computer directories, etc. Non-hierarchical names like sequential numbering are also in use, for example in stellar catalogs. Sequential schemes often lead to ambiguities and confusion, the worst example being the designations of cluster stars by their numbers in several non-overlapping lists. The WDS (Mason et al. 2003) also uses a sequential system (letters A, B, C, etc.) for designating visual components.

Unfortunately, it is not possible to maintain consistent hierarchical designations because hierarchy of multiple stars is not exactly known. There is no problem when some component is found to be a close binary: in this case, the designations are simply extended to the next level. However, when new components are discovered at intermediate levels, as shown below, it is not possible to maintain consistent hierarchical designations without modifying existing designations, which is forbidden. So, hierarchical principles for designating components can be used only as a general guideline; they are bound to fail in some cases and then a work-around will be necessary.

Whatever designation system is eventually adopted, it cannot be expected to work "by itself". So, a centralized database that keeps track of known components and their designations is necessary. We hope that a web-based catalog like WMC can play the role of a "clearing house" for component designations. By developing a set of rules (designation system), we simplify the task of naming new components and, hopefully, diminish confusion, but it is still essential to have a single clearing house. 


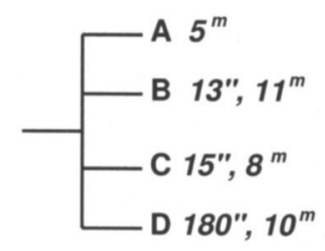

1830: STF 653

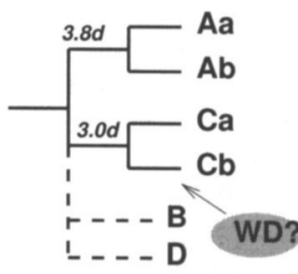

1997: HR 1706

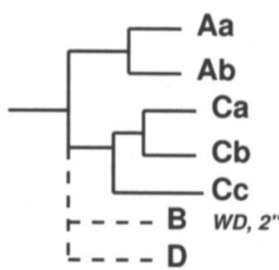

2001

\begin{tabular}{|l|l|}
\hline System & Parent \\
\hline A,C & root \\
Aa,Ab & A \\
Cab,Cc & C \\
Ca,Cb & Cab \\
A,B & Opt. \\
A,D & Opt. \\
\hline
\end{tabular}

Figure 2. The history of 14 Aur = HR 1706 multiple system and its hierarchy

\section{Example: HR 1706}

A $5^{\text {th }}$ magnitude star 14 Aur $=$ HR 1706 began its history as a quadruple, with 4 components A, B, C, D listed in 1830 by W. Struve (Fig. 2). Later it became evident that $\mathrm{B}$ and $\mathrm{D}$ are optical, whereas $\mathrm{A}$ and $\mathrm{C}$ share common proper motion. A was found to be a 3.8-day spectroscopic binary, $\mathrm{C}$ also turned out to be a spectroscopic binary with 3 -day period (Tokovinin 1997a). This star is among the brightest in the sky in the far UV region of the spectrum because it hosts a young and hot white dwarf spatially coincident with C. Subsequent imaging with HST revealed that this WD is not identical to the spectroscopic secondary $\mathrm{Cb}$, as one might expect, but is instead $2^{\prime \prime}$ away from $\mathrm{C}$ (Barstow et al. 2001). The whole system is thus quintuple.

The three panels in Fig. 2 reflect progress of our knowledge of HR 1706. As the structure of many other stars is still not known (or known at the level of Struve), a designation system has to be historical, being able to follow our improving knowledge. There is no sense in attempting a major revision of all designations now because, as time goes by, "new" designations become as obsolete as the current ones.

The component designations in Fig. 2 conform to the proposed WMC scheme and do not change as new components are discovered. This is why the WD is designated as Cc: a consistent implementation of hierarchical principle would require it to be $\mathrm{Cb}$, the spectroscopic pair being $\mathrm{Ca} 1$ and $\mathrm{Ca} 2$, but in this case there would be a confusion with spectroscopic secondary which was $\mathrm{Cb}$ before the WD was discovered. In this case it is better to deviate from strictly hierarchical names rather than create confusion.

If we happened to observe this system few million years ago, the Cc could be a visible star, maybe even the brightest component in the system. In this case it would be already designated by a letter in the WDS, say E (the next available letter).

Table 1 illustrates three available options in the application of the WMC scheme to HR $1706 \mathrm{C}$, all likely to be encountered in practice. Designation 1 - consistent hierarchical names - would be appropriate if all components were discovered jointly and were designated for the first time. Designation 2 reflects the actual discovery history and is the correct option. Designation 3 would apply if the WD were identified as a visual component. The difference between 
Table 1. Application of the WMC designation scheme to HR $1706 \mathrm{C}$

\begin{tabular}{ll|lll}
\hline Type & Object & Desig. 1 & Desig. 2 & Desig. 3 \\
\hline Component & Spectroscopic primary & $\mathrm{Ca} 1$ & $\mathrm{Ca}$ & $\mathrm{Ca}$ \\
Component & Spectroscopic secondary & $\mathrm{Ca} 2$ & $\mathrm{Cb}$ & $\mathrm{Cb}$ \\
Component & White dwarf & $\mathrm{Cb}$ & $\mathrm{Cc}$ & $\mathrm{E}$ \\
Super-component & Spectroscopic binary & $\mathrm{Ca}$ & $\mathrm{Cab}$ & $\mathrm{C}$ \\
Super-component & SB+WD & $\mathrm{C}$ & $\mathrm{C}$ & $\mathrm{CE}$ \\
System & Spectroscopic binary & $\mathrm{Ca} 1, \mathrm{Ca} 2$ & $\mathrm{Ca}, \mathrm{Cb}$ & $\mathrm{Ca}, \mathrm{Cb}$ \\
System & WD orbit & $\mathrm{Ca}, \mathrm{Cb}$ & $\mathrm{Cab}, \mathrm{Cc}$ & $\mathrm{C}, \mathrm{E}$ \\
System & AC orbit & $\mathrm{A}, \mathrm{C}$ & $\mathrm{A}, \mathrm{C}$ & $\mathrm{A}, \mathrm{CE}$ \\
\hline
\end{tabular}

designations 2 and 3 is subtle: in the first case the super-component $\mathrm{C}$ includes all 3 stars, in the second case it refers only to the close spectroscopic pair. As the WD gives no appreciable visible light and thus the existing data on $\mathrm{C}$ refer practically to the spectroscopic pair, it might appear irrelevant which of the options 2 and 3 is selected. However, the last line in Table 1 - designation of the wide system AC - shows that this is not so. If we do not want to change existing records in the catalogs, then option 3 is not acceptable because it would imply changing A,C into A,CE after the discovery of the WD.

The option 2 designates the super-component as Cab. This is only one possibility adopted here. No rules for super-component designations are formulated as yet, although WMC uses implicitly joint letters like BC to designate supercomponents formed by close visual pairs. Current WMC implementation does not make any difference between super-components and systems by omitting sometimes the comma between component's designation. This should be rectified in the future: super-components and systems are different physical entities, they are associated with different types of data. Moreover, a consistent designation of components and super-components permits easy and flexible description of hierarchy, as shown below.

\section{Coding the hierarchies}

If hierarchical designations could be made strict and consistent, there would be no need for separate coding of the multiple-system's hierarchy. Unfortunately, this is not possible, hence we must describe hierarchy separately from component designations and change it flexibly as our knowledge progresses. Another reason to keep hierarchy separate is that in many cases the hierarchy is not established yet and may even be subject to interpretation: nothing forbids to consider Mercury as a close companion to the Sun in a wide system Sun-Jupiter.

Stable multiple systems of stars are hierarchical and can be de-composed in several approximately Keplerian sub-systems. These structures are described by binary trees and can be represented in graphical form as in Figs. 1,2. There are other ways of coding hierarchies. For example, D. Evans proposed bracket notation: the triple in Fig. 1 would be written as a formula $(\mathrm{A},(\mathrm{B}, \mathrm{C}))$. In the Multiple Star Catalog (Tokovinin 1997b) the hierarchical levels are coded as 
sequences of digits: 1 for the upper level A,BC and 12 for the second level B,C. This scheme is descriptive and formal enough to be used in automatic analysis of the multiple-star structure (e.g. for computing the mass of a super-component by summing the masses of all its constituents).

If a consistent system for designating components and super-components is developed and implemented, the best and most flexible way to code the hierarchy is by reference to a parent. Such structures are known in mathematics as chained lists are are formally equivalent to graphs. A special column in the catalog would list the designation of parent - a super-component that hosts any given sub-system. The widest system is the "root" of hierarchy. A collection of records for any given multiple system specifies its hierarchy in a unique way. As new components are discovered, the "parent" column can be changed, but the designations of old components and super-components remain fixed. Application of this idea to HR 1706 is shown in Fig 2.

References can be used for coding hierarchies of planetary systems as well. For example, in the Solar system all orbits of major planets will have root or Sun as parent because they are all at the highest hierarchy level. The EarthMoon orbit will have Earth as parent, all satellites of Jupiter will be linked to Jupiter, etc. Here we avoided the notion of super-components, replacing them with primary bodies of corresponding systems. This is a valid possibility, although some confusion is created when Earth as a physical body has the same designation as the Earth-Moon system orbiting the Sun. This example shows that the concepts of super-components, systems and hierarchy coding are useful for any designations, even for the existing ones.

It is recommended to add a "parent" column to the WMC. It will contain the designation of parent super-component or will remain blank if hierarchy is not known yet. Optical components can be marked as such in the same column.

\section{References}

Barstow, M.A., Bond, H.E., Burleigh, M.R., Holberg, J.B., 2001 MNRAS, 322, 891

Hartkopf, W.I., \& Mason, B. D. Addressing confusion in double star nomenclature: The Washington Multiplicity Catalog, 2004, IAU Colloq. 191, THE ENVIRONMENTS AND EVOLUTION OF DOUBLE AND MULTIPLE STARS, Mérida, Yucatán, México, C. Allen and C. Scarfe, editors, RevMexAA(SC), in press ${ }^{19}$

Mason, B.D., Wycoff, G.L., and Hartkopf, W.I., 2003, The Washington Double Star Catalog 20

Tokovinin, A.A. 1997a A\&AS, 121, 71

Tokovinin, A.A. 1997b A\&AS, 124, 75

\section{Discussion}

DICKEL: Comment: On first glance, it looks like it allows the designation to stay fixed. The changing hierarchy is accomplished by the links to the "parent."

\footnotetext{
${ }^{19}$ see http://ad.usno.navy.mil/wds/newwds.html

${ }^{20}$ see http://ad.usno.navy.mil/wds/wds.html, and Mason et al. 2001, AJ, 122, 3466.
} 


\section{TOKOVININ: Yes.}

SCARFE: For a long time we didn't know to which of the visible pair the third component was attached in the case of 16 Cygni. This is a problem for any scheme.

TOKOVININ: It would not be a problem for sequential numbering. As to the "hierarchical naming," there are two options: i) call it "C" (if A and B are visual components) and define its relation to either $\mathrm{A}$ or $\mathrm{B}$ by links when it becomes known; ii) use provisional designations ?a and ?b for the spectroscopic components.

SCARFE: What is the significance of the $1,11,121,12$ notation, in parallel with Aab, Cab etc.?

TOKOVININ: The numbers like ' 121 ' in MSC are used to code the hierarchy. This number means that the system is a binary primary component of a wider pair which, in turn, is a secondary in a still wider system. The designations like 'Cab' are not sufficient to describe the hierarchy, as we all know.

OSWALT: How do you handle optical pair designations? When a new system is found but the hierarchy isn't evident, how do you assign a designation?

TOKOVININ: Hierarchy is coded separately from component designation. I suggest that any new component be designated on the basis of partially known hierarchy, or by an upper-case letter if it is a "visual" distant component of unknown physical relation.

HARTKOPF: In your case of a pair where one of the two components is an $\mathrm{SB}$, you suggest the designation "?" be used in place of "A" or " $\mathrm{B}$ ". This would seem to lose information in the case where, for example, you know that the SB belongs to either the A or B component of a triple.

TOKOVININ: The new SB will have the same WMC identifier as the visual binary, so it will be clear that the spectroscopic components are either $\mathrm{A}$ or $\mathrm{B}$. 